\title{
Les mots et les sources
}

Words and sources

\section{Ronan Calvez}

\section{OpenEdition}

\author{
Journals
}

Édition électronique

URL : https://journals.openedition.org/lbl/1752

DOI : $10.4000 / \mathrm{lbl} .1752$

ISSN : 2727-9383

\section{Éditeur}

Université de Bretagne Occidentale - UBO

\section{Édition imprimée}

Date de publication : 1 février 2013

Pagination : 81-110

ISBN : 979-10-92331-00-4

ISSN : 1270-2412

\section{Référence électronique}

Ronan Calvez, "Les mots et les sources », La Bretagne Linguistique [En ligne], 17 | 2013, mis en ligne le 01 mai 2021, consulté le 22 mai 2021. URL : http://journals.openedition.org//bl/1752 ; DOI : https:// doi.org/10.4000/lbl.1752

\section{(c) (1)}

La Bretagne Linguistique est mise à disposition selon les termes de la Licence Creative Commons Attribution 4.0 International. 


\section{Ronan CALVEZ*}

\section{Les mots et les sources}

Je n'ai du tout pas de peine à me persuader, je dirai même que je suis convaincu que l'excrément de l'homme a pris son nom du breton, puisque ce nom est écrit dans un vieux Dictionnaire françs-latin. Stronc de chien, stercus caninum. Mais je ne conçois pas quelle fausse pudeur obligeoit $\mathrm{D}[\mathrm{om}]$. $\mathrm{P}$ [elletier]. d'user de périphrase et de circonlocution dans un dictionnaire, pour désigner l'excrément de l'homme, sans oser se servir du mot propre qui convient, tant pour exprimer exactement la chose, que pour dévoiler clairement la pensée de l'auteur

Pour moi je veux nommer la chose par son nom

J'appelle un chat un chat et le stronc un etron ${ }^{1}$.

S'il s'agissoit de composer une langue nouvelle, les inventeurs se piqueroient sans doute d'en faire un tout bien compassé et bien régulier, dont toutes les parties seroient dans une harmonie parfaite et ne laisseroient rien à désirer ; mais le but d'un dictionnaire doit être de nous présenter la langue telle qu'on la parle, sans prétendre l'asservir, sous prétexte de régularité, à de nouveaux systèmes de reforme, auxquels la langue bretonne en particulier repugne toujours, et contre lesquels elle s'est constamment roidie, malgré les efforts des novateurs ${ }^{2}$.

* Professeur de celtique, CRBC (EA 4451), UBO/ueb

1. VII, 873, sous «stronc», à la question de savoir si ce dernier mot désigne ou non l'excrément.

2. V, 457, sous «miret», «ou, pour mieux dire mira». 
ans son grand œuvre, Coëtanlem cite avec abondance le fabuliste La Fontaine ${ }^{3}$. Plongé dans les pages du dictionnaire à la recherche des sources bretonnes de l'auteur, je me suis parfois demandé si la montagne n'allait pas accoucher d'une souris. En effet, au premier abord, la récolte peut sembler bien maigre. Néanmoins, entre les lignes, se laisse entendre assez précisément ce que parler bretonet du breton - veut dire pour le sieur de Coëtanlem. Car c'est bien d'oralité dont il est question ici : alors que les sources françaises et latines du lexicographe sont livresques, ses sources bretonnes sont orales.

\section{*}

Lorsqu' on lit le volumineux dictionnaire, on voit très clairement se dessiner deux types de sources qui témoignent de deux types de représentations. Une fois mise de côté la Bible qui est citée, certes, mais sans abondance, la première source de Coëtanlem est classique, latine et française, la deuxième est bretonne.

En effet, les citations latines abondent dans le texte et les auteurs les plus cités sont sans doute Virgile - avec les Géorgiques ${ }^{4}$, les Bucoliques $^{5}$ ou l'Énéide ${ }^{6}$-, Ovide - ses Métamorphoses ${ }^{7}$ et son Art d'aimer $^{8}$-, Horace - avec les Satires ${ }^{9}$ et l'Art poétique ${ }^{10}$-, Martial et ses Épigrammes ${ }^{11}$ ou encore Juvénal et ses Satires ${ }^{12}$. On trouve aussi sous la plume de Coëtanlem des références à Lucrèce, pour son De la Nature ${ }^{13}$, ce qui peut sembler plus curieux mais qui s'explique sans doute par son admiration pour La Fontaine, grand lecteur du premier.

\footnotetext{
3. Voir son ode au style et à la morale de la Fontaine (IV, 568).

4. Par exemple : II, 847 ; II, 894 ; III, 30-31.

5. Par exemple : II, 944 ; III, 340.

6. Par exemple : II, 11 ; II. 853.

7. Par exemple : II, 922.

8. Par exemple : I, 66-67; II, 848.

9. Par exemple : II, 945.

10. Par exemple : I, 118 ; II, 1040.

11. Par exemple : II, 12 ; III, 597-598; III, 838.

12. Par exemple : IV. 324.

13. Par exemple : III, 1085 ; III, 1124.
} 
En effet, en français, Coëtanlem a également ses classiques, au premier rang desquels figure, sans conteste, La Fontaine, ses Fables ${ }^{14}$ mais aussi ses Contes $^{15}$. On trouve également des références à Racine - Andromaque ${ }^{16}$, Les Plaideurs ${ }^{17}$ ou Phèdre ${ }^{18}$-, Corneille Le menteur ${ }^{19}$ ou Attila ${ }^{20}$-, Boileau - ses Satires ${ }^{21}$ et son Art poétique $^{22}$-, Philippe Destouches - Le philosophe marié ${ }^{23}$ ou encore L'enfant gâté ${ }^{24}$. Enfin, Coëtanlem puise également dans des recueils de poésie, par exemple la Bibliothèque poëtique, ou nouveau choix des plus belles pièces de vers en tout genre, depuis Marot jusqu'aux Poëtes de nos jours ${ }^{25}$. Tous ces auteurs illustrent les démonstrations du lexicographe et ils lui servent de caution - on peut sans doute y lire également une trace profonde de l'enseignement classique dont il a bénéficié : «à l'égard de la crosse, je n'ai pas oublié que j'y ai joué autrefois étant au collège ${ }^{26} \gg$. Ainsi, va-t-il s'appuyer sur Virgile pour montrer l'ancienneté de telle ou telle pratique ainsi que de tel ou tel propos «chez nos paysans» - «Nos païsans disent encore que les pierres sont les os de la terre ezkern an douar. Les anciens ont eu la même idée et leurs poëtes et leurs mythologistes en ont tiré bon $\operatorname{parti}^{27} \gg$.

Tous ces auteurs cités témoignent de sa connaissance de la littérature classique mais également de l'étendue de sa bibliothèque. Pour lui, ils représentent avant tout la sagesse antique ou classique pluriséculaire, complétée par des ouvrages plus récents qui consti-

14. Par exemple : I, 592.

15. III, 448.

16. II, 667.

17. VI, 620.

18. VIII, 466.

19. III, 563 ; VIII, 466.

20. III, 957 bis.

21. II, 909.

22. II, $462 ;$ IV, 267.

23. III, 525.

24. III, 623.

25. Le tome premier de ce recueil est publié en 1745. Coëtanlem cite plus particulièrement les œuvres du «Chevalier de Cailly ou d'Aceilly» (voir par exemple : II, 393 ; III, 256).

26. VIII, 349, sous «tinta». Bien entendu, il faudrait mener plus avant des recherches sur ces années de formation, sous l'Ancien Régime.

27. I, 158, sous «askorn». 
tuent la source érudite. En effet, aux côtés des celtomanes - Pezron ${ }^{28}$, Cambry ${ }^{29}$, La Tour d'Auvergne ${ }^{30}$ ou Le Brigant ${ }^{31}-$, on trouve sous sa plume des références à des ouvrages qui ont en commun de diffuser et de vulgariser les idées des Lumières et le relativisme, mais aussi et peut-être surtout de développer le goût pour la connaissance et l'encyclopédisme - le Traité de l'opinion ou Mémoires pour servir à l'histoire de l'esprit humain, de Gilbert-Charles Le Gendre ${ }^{32}$; le Manuel du naturaliste ${ }^{33}$, ouvrage de botanique «dédié à M. de Buffon"); le Dictionnaire oeconomique : contenant l'art de faire valoir les terres et de mettre à profit les endroits les plus stériles [...], de Noël Chomel ${ }^{34}$; Le spectacle de la nature ${ }^{35}$, de l'abbé Pluche...

Néanmoins, en plus de cette source française et latine, littéraire et érudite, il existe une source bretonne. Bien entendu, Coëtanlem cite abondamment les dictionnaires de ses prédécesseurs mais, on s'en doute, ce n'est pas ce qui est le plus intéressant. Il mentionne aussi des proverbes, trouvés chez Grégoire de Rostrenen notamment, mais qu'il semble connaître ${ }^{36}$ ou bien également des couplets

28. Pour Antiquité de la Nation et de la langue des Celtes, autrement appeléz Gaulois, 1703 (VIII, 75).

29. Pour son Voyage dans le Finsitère ou état de ce département en 1794 et 1795 (VI, 533) ou ses Monumens celtiques, imprimé en 1805 (II, 803 ; IV, 1062 ; VI, 905).

30. Pour Origines gauloises, celles des plus anciens peuples de l'Europe, puisées dans leur vraie source, ou recherches sur la langue, l'origine et les antiquités des Celto-Bretons de l'Armorique (...), an V (VI, 904).

31. Pour sa Dissertation adressée aux académies savantes de l'Europe, sur un peuple celte, nommé Brigantes, ou Brigants, 1762 (VI, 583).

32. Six volumes imprimés à compter de 1735 (par exemple : II, 316 ; II, 319).

33. Imprimé en 1771 et rédigé par $\mathrm{D}^{* * *}, \mathrm{M}^{* * *}$ (par exemple : II, 801 ; II, 1077).

34. La première édition est de 1709 mais il cite l'édition de 1767 en 3 volumes (III, 429).

35. Le spectacle de la nature, ou Entretiens sur les particularités de l'histoire naturelle qui ont paru les plus propres à rendre les jeunes gens curieux et à leur former l'esprit: la première édition de cet ouvrage, qui connait un grand succès dans toute l'Europe, est de 1732 (I, 672 ; III, 536).

36. Par exemple : I, 236, sous «bac'h»; I, 513, sous «boursavelec»; III, 632 sous «ghin»; III, 635, sous «ghis»; V, 304, sous «merçz»; VI, 57, sous «paner»; VI, 77, sous «paour»; VI, 414, sous «pinwidic»; VI, 729, sous «raz». Mais il ne manque pas également de prendre le Père Grégoire en défaut : «Je m’etonne que ce Père qui rapporte presque tous les plats quolibets qu'il a entendus, et qu'il 
de chansons dont il dit se rappeler - certaines sont en français ${ }^{37}$. Ces sources souvent inédites méritent d'être citées car, même si certaines chansons relèvent de l'amusement ${ }^{38}$ ou bien sont des

nous donne pour des proverbes, n'ait pas cité ce rebus ou ce dit-on vulgaire dont on se sert communément pour justifier le boire et le manger ou pour y exciter : An tam hag al loum a zalch an den en he bloum (ou bien) An tam hag al lôm a zalc'h an den en he blôm, ce qui veut dire à la lettre, Le morceau et la goute maintiennent l'homme dans son à-plomb, pour faire entendre que ce sont là les soutiens de la vie» (IV, 1152-1153, sous «lom»); «D.P. parlant de mil vecher, qui est de mille métiers, $\&^{c}$ me rappelle un dit-on assez vulgaire qu'on ne manque pas de citer toutes les fois qu'il est question de ces sortes de personnes qui se vantent de posséder une infinité de talents, qui forment quantité d'entreprises, qui se mèlent de mille affaires. Il est conçu ainsi : Daniel mil micher a varwas gand an naounn, ce qui veut dire Daniel aux mille métiers mourut de faim» (V, 211, sous «mecher»). Il donne quelques proverbes en français, également : II, 445 , sous «cornighell».

37. Par exemple : VI, 712-713 ; VII, 567 ; VIII, 83 ; VIII, 1004-1006.

38. «J'ai entendu chanter une gavotte où l'on disoit :

Dont a eure da Ghæ-ahes

Diloezr ha divotes.

Ce qui signifie : il vint à Carhaix sans bas et sans soulier, c'est-à-dire qu'il arriva nuds pieds.» (II, 1053, sous «divotes», qui ne figure pas dans le dictionnaire de Le Pelletier).

«Le possessif Mastrouilleg, plein d'ordures, est employé comme epithète d'un gros chien dans une chanson que j'ai souvent entendu chanter.

Ar moc'h, ar zaout, hag ar c'hezeg,

A zouje ar c'hi mastrouilleg, $\&^{c}$

ce qui veut dire : les cochons, les vaches et les chevaux

redoutoient le chien chargé d'ordures, $\&^{\mathrm{c}} »(\mathrm{~V}, 198$, sous «mastrouill ». Il cite ce même extrait sous «moustrouill», V, 646).

«Les Bret. pour désigner les feuilles vertes de plusieurs plantes font souvent précéder le nom qui leur est propre du mot cawl, qui signifie choux : cawlouignon, cawl-pour $\&^{c}$. et j'ai entendu un vieux fragment de chanson qui disoit :

Batara hag he vreg

En hem gannas nos Nedeleg

abalamour d'ar c'hawl pour

a voa ken tanao ha dour.

Ce qui veut dire que Batara et sa femme se battirent la nuit / de Noël à cause des choux-porreaux qui étoient aussi clairs que de l'eau; ou à cause des feuilles de porreaux $\&^{\mathrm{c}}$ » $(\mathrm{VI}, 545-546)$.

"Nos diction. expriment bouder en latin par obmurmurare ; mais cela ne rend pas exactement notre Mouza, bouder, et proprement faire la moue, témoigner son humeur ou son mécontentement par cette vilaine grimace des lèvres, sans bruit, sans murmure, sans parler, sans dire mot, comme dit la vieille chanson : 
classiques ${ }^{39}$, d'autres éclairent d'un nouveau jour l'histoire littéraire et sociolinguistique du breton. Elles disent, en creux, des pratiques sociales dont la langue bretonne est une part langagière essentielle.

Je me rappelle aussi d'un couplet de chanson où Baudreou et Botou prenn sont également joints ensemble. Cette chanson avoit été faite à l'occasion d'un paisan devenu riche et glorieux, et qui avoit orné son chapeau d'un grand plumet. On la lui chanta à la procession du sacre, où il étoit sous les armes. Voici ce couplet, Air du Pange Lingua :

Thomas Cozten, glorius oc'h,

Ar Bêd oll a gomps ac'hanoc'h, abalamour d'ho plumachenn

a so kehit ac eur scubælenn,

Peleac'h ez ma ho coz juppenn, ho paudreou, ho poutou prenn?

Ce qui veut dire : Thomas Cozten, vous êtes glorieux, tout le monde parle de vous à cause de votre plumet qui est aussi long qu'un balai. Où sont votre vieux pourpoint, vos gamaches ou vos guêtres et vos souliers de bois ou vos sabots?

J'ai connu le petit fils de ce Thomas Cozten, mais je crois sa race éteinte. Sans cela je n'aurois pas pris la liberté de transcrire ici ce petit couplet satyrique qui auroit pu faire de la peine à ses descendants ${ }^{40}$.

Ce couplet témoigne de la volonté des riches paysans d'intégrer, réellement ou symboliquement, la noblesse et de l'imiter, au niveau

Mouzet ew ïan, ne lavar gher,

Troet he benn oc'h ar vogher. $\&^{c}$.

Ce qui veut dire littéralement : jean est boudé, il ne dit mot, sa tête est tournée vers la muraille.» $(\mathrm{V}, 634$, sous «mousa»).

39. «Quoiqu'il en soit le mot Hirvoudi s'applique quelquefois aux bêtes, aussi bien qu'aux hommes; et je me rappelle d'avoir entendu chanter un couplet qui se terminoit de la sorte :

Evel an durzunell, pa zeu da goll he phar,

Ne ra nemet hirvoudi pelloc'h war an douar.

Ce qui veut dire : comme la tourterelle, lorsqu'elle vient à perdre son pareil (c'est-à-dire lorsqu'elle perd son mâle) ne fait plus désormais que gémir sur la terre.» (IV, 149, sous «hirvout»).

40. I, 312 sous «baudreou». 
vestimentaire comme au niveau de l'habitat ${ }^{41}$. Ce désir se retrouve dans deux autres chansons citées par Coëtanlem.

Dans ce paiis, nous appelons le marchand de balais : Scubælennäer, pl. Scubælennærrien. fémin. sing. Scubælennæres, pluriel Scubælennæreset. Ce Scubælennäer, est, comme on voit, un dérivé du sing. défini Scubælenn; et je l'ai trouvé dans une vieille chanson satirique, où on déclame contre les artisans qui quittent leurs habits simples et modestes, pour se mettre à la mode des grands. Voici le couplet où il est question de Scubælennäer :

Ne deus merc'h scubælennaer,

Pautr merchaussi, na marmiton,

Lazer moc'h, na pillaouäer

N'a zougont holl ar c'hrepon :

Ar chass a staut gand an donjer,

Ha darn a gri holelard,

ô velet merc'h eur c'hemener,

hag hi gwisket e brocard ;

he zud côz a hed hô amzer

a veve dioc'h ar bombard.

Ce qui veut dire : il n'est pas de fille de marchand de balais, de valet d'écurie, ni de marmiton, de tueur de cochons, de marchands de chiffons qui ne portent toutes le crêpon. Les chiens pissent de rancune, et une partie crie au lard (mardi-gras) en voyant la fille d'un tailleur habillée ou vêtue de brocard. De tout temps ses ancêtres vivoient de la bombarde, c'est-à-dire qu'ils étoient joueurs d'instruments. Le mot Scubælenn d'où est tiré Scubælennäer se trouve aussi dans une autre chanson satirique, dont j'ai inséré un couplet dans mes remarques sur Baudreou ${ }^{42}$.

Le mot viltançç est aussi employé dans une vieille chanson satyrique contre les femmes du peuple qui adoptoient les modes des Dames. Le dernier couplet étoit conçu à-peu-près en ces termes :

Ma teuffe hon tud ancien

a newez flamm war ar bed,

e clanfent gant ar gredien ;

41. Voir Sébastien CARNEY, «Les riches maisons paysannes du Léon aux XVIIe et XVIII' siècles ou les palais des rois-paysans ", Annales de Bretagne et des Pays de l'Ouest, t. 107, n 3, 2000, p. 57-86.

42. VII, 344-345, sous «scub». 


\begin{abstract}
e vent meurbet souezet, ô welet mascaradennou ô caout an assurançç, war ô phenn jobilinennou, da zônet en hô phresançç, ne rofent nemet mallozziou d'ar seurt pennou a viltançç.
\end{abstract}

Il est à remarquer que ces jobelines sont à présent abandonnées aux paisannes ${ }^{43}$.

Même si ces critiques des paysans ou artisans parvenus ne sont peut-être pas issues d'une plume noble, les deux couplets qui suivent fleurent bon la satire nobiliaire et témoignent, eux aussi, d'une exacerbation de la clôture - qui détermine les rapports des membres de la noblesse aux autres ${ }^{44}$, et de la connivence - qui régit les rapports entre ces mêmes membres ${ }^{45}$ :

j'ai encore trouvé Discoghell dans un ancien couplet de chanson, où il étoit employé figurément et malgré les sens divers et les origines différentes de Discoghella, le Discoghell de la chanson pourroit se concilier un peu avec l'un et l'autre. C'etoit une chanson satyrique contre les parvenus et surtout contre leurs femmes, qui quittoient la quenouille et le fuseau pour s'occuper de toilette, ou qui affectoient de prendre de grands tons, de grands airs, de riches parures à l'imitation de la noblesse à laquelle seule il étoit permis de porter de la soie, d'après les loix somptuaires de nos anciens rois. Quoiqu'il en soit, voici ce couplet, afin que chacun en puisse juger.

43. VIII, 859, sous «viltanç». Voici une traduction de la chanson : «Si nos ancêtres revenaient / De nouveau sur la terre, / Ils s'évanouiraient de stupeur, / Ils seraient très étonnés / À la vue de ces mascarades / Qui ont l'assurance, / Sur la tête, des jobelines, / De venir en leur présence. / Ils ne feraient que maudire / De telles têtes d'infamie». Sauf indication contraire, les traductions sont de moi.

44. «Je crois que partout le peuple mange de la vache aussi bien qu'en Bretagne, et qu'en Bretagne comme ailleurs le bœuf est reservé pour la table des riches» (I, 663 , sous «buoc'h»); à propos d'un «regal» nommé «Panes Goudez», composé de lard et de panais : il n'a «jamais mangé de ce regal vanté par nos campagnards, dont l'estomach digère mieux que le mien» (II, 209, sous «caudet»).

45. Voir Antoine LiLTI, «Mondanité et Révolution : les hommes de lettres et la sociabilité mondaine à la fin du XVIII' siècle», dans Philippe BOURDIN et Jean-Luc ChAPPEY, Réseaux \& sociabilité littéraire en Révolution, Presses Universitaires Blaise-Pascal, Collection Histoires croisées, 2007, p. 49. 
Etrezoc'h, noblanç discoghell,
a so sawet a wal raçç,
neb o caffe dindan cebell
a dleffe brêwi o façç
List ar c'hebell gant an noblançc
Pere a ell o doughenn,
Deoc'h-hu ne deo ket dismegançc
Beza coeffet e lienn

Ne vot ket gwaz dighemmeret gant o kerent kigherrienn ${ }^{46}$.

On voit bien que Discoghell est un adjectif ou une epithète dont on qualifie cette noblesse parvenue, et qu'on pourroit traduire ce couplet ainsi : Entre vous autres, noblesse equenouillée (en adoptant la définition de D.P.) ou bien entre vous autres, noblesse imitatrice des grands tons, qui êtes issue d'une méchante race, quiconque vous trouveroit sous le chaperon devroit vous casser la figure ; Laissez les chaperons à la noblesse (c'est-à-dire aux vrais nobles) qui peuvent bien en porter; Pour vous ce n'est pas deshonneur d'etre coëffées en toile : vous n'en serez pas plus mal accueillis de vos parents bouchers ${ }^{47}$.

Au mot Discoghell, j'ai inséré un couplet où ce mot étoit inséré. Le mot Drouin se trouve aussi dans le couplet suivant de cette vieille chanson.

Livirit dign, traou diböellet,

Demesellet a wall chanç, pe gant piou oc'hu bet gwallet, d'ho transformi e noblanç?

ho re nessa so carserrienn ha dougherrien an drouin, spazerrienn moc'h ha crouezrienn ha gwiaderrienn ballin, pillaouerrienn, trafikerrienn d'ar pez a chomm er gribin.

Ce qui signifie : Dites-moi, etres sans retenue, demoiselles de male-chance, par qui avez-vous été engrossées pour vous transformer en noblesse ? Vos proches sont des vuidangeurs et des porteurs de drouine (ou des chaudronniers) des saneurs de porcs,

46. En marge, Coëtanlem écrit ceci : «Cette derniere ligne devoit faire deux vers, mais la rime étoit en défaut.»

47. II. 967-968 sous «discoghella». 
des faiseurs de cribles (ou des vanniers) des tisserands de ballins, des chiffonniers, des traficants de ce qui reste dans le seran (c'est-à-dire des marchands d'etoupe $)^{48}$.

De même, la chanson qui suit, innocemment citée par Coëtanlem, me semble plus ambiguë qu'il le laisse entendre :

Jai entendu au sujet d'un coq un fragment de chanson bretonne qui paroissoit assez jolie, mais je n'en connois que le commencement. Le voici :

Allas! Siwas marw et zew va c'hog,

Caezr en deveus bet beza rog,

Collet en n'eus he grog :

E Lesneven ew decedet,

Cals a ïer zo intanvezet,

Ne allont mwy dezwi, $\&^{\text {c. }}$

c'est-à-dire en $\mathrm{f}^{\mathrm{t}}$

hélas ! malheureusement mon coq est mort,

il a eu beau être arrogant,

il a perdu son croc :

il est décédé à Lesneven

Bien des poules sont veuves,

Elles ne peuvent plus pondre $\&^{\text {c. }} 49$

Ces sources sont intéressantes car elles témoignent de sa connaissance de productions bretonnes qui, semble-t-il, n'ont laissé aucune trace par ailleurs et n'ont, en tous les cas, pas été imprimées. Dans les extraits que j'ai donnés, je vois la marque du breton mondain : le coq introduit un double sens; la critique de la noblesse parvenue est assurément écrite par des nobles. De même, dans une remarque qui suit la définition du verbe «scrapat», Coëtanlem présente une pratique sociale qui devait avoir peu cours dans les fermes de Poullfaouig ou Keribilbeus mais qui est pourtant dite en breton :

On emploie également le verbe Scrabat ou son composé Discrabat pour dire : faire la revérence ; et le dérivé Scraber ou Discraber, pour désigner celui qui fait la revérence ou des révérences. Le P.G. au mot revérence, salut respectueux a mis aussi

48. II, 1186 sous «drouin».

49. II, 374 sous «cockilloc». 
Discrab, pl. Discrabou : faire la revérence, Discrabat ; et sur Revérentieux, il a mis un Discraber bras, pl. Discraberyen vras. Comme les femmes se contentent de fléchir les jarrets, sans avoir l'air de grater la terre, leur revérence s'appelle Stou, que l'on trouvera ci-après en son rang ${ }^{50}$.

Il en va sans doute de même pour la mode des talons, qui devait être peu pratiquée par celles qui fréquentaient les chemins creux :

Nous donnons à la hausse artificielle que les femmes font mettre à leurs souliers, afin de paroître plus grandes, le nom de Cornel ou Corniel-botes. V. ce mot. Et mettre de telles hausses Corniela Botou, Boutou ou Bouteïer. On fait des hausses de différentes matières, mais on prétendoit que celles de corne duroient plus long-temps. Au reste les femmes commencent à se défaire de cette mode aussi gênante que bizarre, sauf à la rependre encore quelque jour ${ }^{51}$.

Enfin, sous «truez», il donne un exemple admirable de toute la distinction sociale qui sied à une Dame, bonne pour ses pauvres.

De là ces expressions si fréquentes : Ar paour kæs-ze a zô en eur stad truezus, Ce pauvre malheureux là est dans un etat pitoyable. Ann itroun a zô truezus ewit an dud ezommeg, La Dame est pitoyable envers les indigents, ou envers les personnes qui sont dans le besoin ${ }^{52}$.

À mon sens, ces derniers exemples témoignent de l'existence d'une pratique mondaine du breton dont il nous offre, bien malgré lui sans doute, un écho déjà lointain. Mais ce n'est pas tout. Ce dictionnaire témoigne de l'étendue des pratiques langagières du sieur de Coëtanlem et de sa bonne connaissance du breton - ou bien plutôt, devrais-je dire, des bretons. En effet, lorsqu'on lit son dictionnaire, on se rend compte que l'érudit nous peint un paysage sociolinguistique assez subtil, dans des teintes nuancées - peut-être même sans le vouloir.

50. VII, 322.

51. II, 136 , sous «carn»

52. VIII, 754. 
De nombreux exemples en fournissent la preuve : Coëtanlem a visiblement une très bonne connaissance des badumes - on trouve d'ailleurs sous sa plume une excellente définition de ce que badume veut dire : «Quant à Trefoet, c'est une epithète par laquelle on désigne le langage de celui qui s'exprime ou qui prononce autrement qu'on ne le fait dans le canton où l'on se trouve; et la même épithète se rend réciproquement par les habitants d'un canton à ceux de l'autre, lorsque la différence de prononciation est très sensible ou assez considérable pour faire impression ${ }^{53} \gg$.

Abôn. Fiente, excrement des animaux. J'ai souvent entendu nos paisans se servir de ce terme qui leur paroît sans doute plus décent et plus honnête que celui de Coc'h. Ils l'emploient surtout pour désigner la fiente de cheval, Abon kesec. Ils ont un autre terme particulier pour désigner la fiente de vache, Bezell saout. Nous le verrons en son rang. Abon, en lat. stercus, ne se trouve pas chez nos lexicographes, mais D.P. parle ci-après de Môn qui semble y avoir quelque rapport ${ }^{54}$.

Lorsqu'il s'agit d'une jument, on ne dit pas Ala dans ce canton, mais on se sert de Trei, qui signifie proprement Tourner, et quelquefois, mais plus rarement Ebeullia ${ }^{55}$.

D.P. peut bien avoir raison dans l'explication qu'il donne d'Awrec, qui est en général Gueret ; mais dans ce canton on donne en particulier le nom d'Awrec à la terre qu'on a labourée pour ensemencer des navets, après avoir tiré le lin. Awriat ou Awria, c'est faire ce travail et Devez Awriat est une journée d'un tel travail ${ }^{56}$.

Quoiqu'il en soit, j'ai entendu quelquefois des marchands de bestiaux se servir du mot ouc'henn, lorsqu'ils parloient des bœufs en général, cependant dans ce païs on se sert plus communément du mot eujenned, pl. régulier d'eujenn ${ }^{57}$.

53. VIII, 560-561, sous «trefoet $»$.

54. I, 14.

55. I, 42, sous «ala ou hala».

56. I, 206, sous «awrec».

57. V, 861, sous «oen». 
Je n'ai pas connu son [à P.G.] fréquentatif Pôkedal en usage ; mais j'ai souvent entendu dire : Beza atô pôk-pôk ; ce qui veut dire à la lettre : etre toujours baise-baise. La répétition du mot est une façon naturelle de marquer le superlatif, ou le fréquentatif ${ }^{58}$.

Pour l'anglais, ou langue anglaise nous disons säosmeg ; parler anglais, saosmegat ${ }^{59}$.

Dans quelqu'uns de nos cantons, j'ai entendu donner à la sève le nom de saotr ; et en parlant des plantes qui montent en graine, on se sert assez généralement du verbe saotra et saotri, participe passif saotret. Ex. Ar panes nag ann irwin ne delont vad pa vezont saotret, Les panais non plus que les navets ne valent rien quand ils sont montés ${ }^{60}$.

Nos lexicographes ne parlent de saour qu'au sens de goût et de saveur ; mais il est sur qu'il n'est pas moins connu dans ce païs au sens d'humidité naturelle ou d'humectation, comme je viens de le dire. Aussi pour faire entendre qu'il a fait assez de pluie, on emploie communément ces expressions : saour awoalc'h a zô brêmâ en douar, il y a maintenant assez d'humidité dans la terre, ou la terre est maintenant assez humectée. Si la sécheresse dure longtemps, on dit : An douar a zô disaour, la terre est sans humidité ${ }^{61}$.

Dans les environs de Morlaix on prononce Sclabez, Sclabeza, Sclabezus ; Disclabez, Disclabeza, $\&^{\text {c. }}$ mais dans le païs de Léon on dit simplement Labez ${ }^{62}$.

Dans ce païs on se sert d'une autre expression qui est pareillement outrée, pour dire que la vache recherche le taureau, Gwenn tarw a zô er vioc'h, ou gant ar vioc'h, mot à mot Race, espèce ou semence de taureau est dans la vache, ou avec la vache ; ce qui exprime beaucoup plus que le désir, qui signifie c'hwant, auquel il auroit fallu s'en tenir ${ }^{63}$.

58. VI, 497, sous «pok».

59. VII, 98, sous «saos».

60 . VII, 111, sous «saotr».

61. VII, 114, sous «saour».

62. VII, 769, sous «stlabez».

63. VIII, 126, sous «taro». 
Dans ce païs l'on dit aussi, en parlant d'un homme qui n'est pas tout-à-fait yvre, mais qui a le cerveau un peu échauffé par les vapeurs du vin, Tomet ew d'ezan̂, (mot à mot, il est échauffé à lui) pour faire entendre que le vin lui a monté à la tête ${ }^{64}$.

Ainsi, de même qu'il fait montre d'une solide connaissance des pratiques agricoles puisée dans ses observations ${ }^{65}$ ou ses lectures de Virgile ou Ovide ${ }^{66}$, met-il en lumière son bel entendement du breton parlé par ses paysans, comme en témoignent également les mots qui ne figuraient pas dans le dictionnaire de Le Pelletier et qu'il intègre au sien ${ }^{67}$. Il a de plus conscience d'une différence de registres au sein du breton parlé lorsqu'il écrit, sous le mot «couihonereh», qu'il est persuadé «que ce mot et plusieurs autres qui lui sont analogues et qui se diversifient de mille manières, ne sont que des termes de jargon qui ne sont de mise dans aucune langue ${ }^{68} 》$ ou encore que les « expressions triviales que chaque charretier ou bouvier forge ou varie, selon son caprice ne valent pas la peine de s'y arrêter ${ }^{69} \gg$. En regard de ce registre paritaire du breton, utilisé par des pairs qui ne sont séparés par aucune limite institutionnelle ou symbolique, Coëtanlem laisse entendre un registre disparitaire, notamment lorsqu'il se pique, dans un petit accès de prétention à l'écriture bretonne et à la distinction littéraire, de traduire la Bible :

Ainsi pour traduire ces paroles de l'Évangile où Pilate rend un hommage public à l'innocence de N.S.J.C. Nihil invenio in hoc homine. Luc. Cap. 23. v. 4. Ego enim non invenio in eo causam.

64. VIII, 396, sous «tom».

65. Voir sa description de la culture du genêt (I, 248 sous «balan») ou du goémon (I, 392), son développement sur le jonc et sur les zones humides (I, 628).

66. Le nom «carr-eaustec» convient pour désigner les « voitures dont on se sert pour transporter le bled, le foin, la paille, qu'on a recueilli pendant le cours de la saison. Le Plaustrum des lat. devoit servir au même usage, puisqu'il est fait de Plous, et cette voiture figuroit aussi dans les fêtes de Cérès, à qui elle étoit en quelque sorte consacrée.

Tardaque eleusina matris volventia Plaustra.

Virg. Georg. Lib. 1. p. 150.»

(«Les chars de la mère d'Eleusis roulent lentement»; III, 30-31, sous «eaust »).

67. À titre d'exemple, voir «teuc' $h »$, VIII, 266, en annexe.

68. II, 492.

69. II, 697, sous «dahalm». 
Joan. C. 18. v 38. je dirois simplement : Ne gavan ket an disterra abec en den-man̂. Rac evitdon-me ne gavan abec ebed eñ-han ${ }^{70}$.

De même, sous l'article «gwalc'h», examine-t-il «gwelc'h», «le lavage ou 1'action de laver»:

Nous avons encore les composés Azgwelc'hi, relaver, laver de plus en plus, va gwelc'hit, va azgwelc'hit, lavez-moi, relavezmoi, Ampliùs lava me, $\&^{\text {c. }}$ Psalm. $51^{71}$. Voici la paraphrase de ce verset :

va gwelc'hit, va azgwelc'hit, grit eur c'hwez d'ho toare :

peur-nættait va ezne, ma vezo adarre

ken agreabl dirazoc'h, eghis pa zortias

diwar font ar vadizziant gant eur zantelælez-vras.

Ce qui veut dire littéralement : Lavez-moi, relavez-moi, faites une buée à votre manière ; achevez de nettoyer mon ame, afin qu'elle soit encore aussi agréable devant vous, comme lorsqu'elle sortit dessus le font du baptême avec une grande sainteté ${ }^{72}$.

Mais il ne se contente pas de la Bible et il traduit également Virgile :

De même pour exprimer le participe quassatus qui signifie furieusement agité, pourchassé, surmené ou mal-mené, je puis me servir de Gwal-gaççet ou Gwal-gasset, qui veut dire la même chose, et qui a l'avantage de présenter également la même racine cass, principe de quassus, dont on a formé quassatus. Je puis donc expliquer de la manière suivante, ce passage de l'Eneïde, où ilionée, l'un des capitaines de la flotte d'Enée demande à Didon la liberté de mettre à sec sur le rivage, afin de les radouber commodément, les vaisseaux troyens chassés par la tempête et entraînés par des vents furieux bien loin des côtes d'Italie, où ils vouloient aborder.

Sens littéral des termes que j'emploie dans ma traduction bret.

qu'il vous plaise, Dame débonnaire; autrement Dame gracieuse, trouvez-bon que nous tirions bout à terre nos vais-

70. «Je ne trouve en cet homme le moindre grief»; «Pour ma part, je ne lui trouve aucun grief». I, 9-10, sous «abec».

71. «Amplius lava me ab iniquitate mea, / et a peccato meo munda me // Lave-moi tout entier de mon mal / et de ma faute purifie-moi» (Psaume 51, 4).

72. III, 966. 
seaux qui ont été rudement secoués, chassés, pourchassés ou mal-menés par les vents déchaînés.

Plijet ganeoc'h, itrôn hegar, ma tennimp à-benn en douar hor listri so bet gwal-gasset gant ann avelou diboëllet.

Virgile est bien plus bref, puisqu'il exprime presque tout cela dans un seul vers, mais du moins je ne crois pas en avoir altéré le sens, et j'ai fidèlement rendu son participe quassatam. quassatam ventis liceat subducere classem ${ }^{73}$. æneïd. L. p. $502^{74}$.

Coëtanlem traduit et, de la sorte, forge un registre disparitaire du breton : dans sa fonction, ce dernier n'est guère différent du breton d'Église qui se lit, par ailleurs, dans ses pages. Ainsi, sous Absolf, Abus, Abusi, écrit-il : «Je ne prétends pas donner ces mots pour vrais bretons, mais le P.G. les a insérés dans son Dict. et de fait ils sont en quelque façon consacrés par l'usage de l'Église ${ }^{75}$.» Ces mots ne semblent pas lui convenir car ils ne sont pas «vrais bretons», et certaines traductions ecclésiastiques sont grossières à ses yeux. Sous l'article «gwelan», il raconte le miracle de saint Paul et des oiseaux de mer qui pillent le blé entreposé :

Ce miracle est encore rappellé sommairement dans l'hymne de vêpres, où l'on dit que $\mathrm{S}^{\text {t. }}$ Paul chassa devant lui, comme un troupeau de moutons, une quantité d'oiseaux qui ravageaient les bleds, et qu'il les renferma dans une cave ; qu'ils n'en sortirent qu'à ses ordres, et qu'ils quitterent enfin ce sol consacré, où ils ne devoient plus remettre les pieds. C'est le sens de ces paroles : alitum turbam sata sublegentem, ut gregem cogit, caveâque claudit, cum jubet, nunquam reditura sanctis exulat agris.

Il existe une traduction bretonne, ou plustôt une imitation assez grossière de cette hymne que $\mathrm{j}$ 'ai entendu chanter aussi sur le même air. Les oiseaux dont il est fait mention dans le latin,

73. «Permets-nous de tirer au sec notre flotte malmenée par la tempête».

74. II, 11, sous «caç̧».

75. I, 15. 
sans les nommer, sont appellés Gweleznet dans le breton. Voici la strophe qui répond à celle que j'ai transcrite cidessus :

E fæçon den̂vet e cundu gweleznet, d'he væstr d'ar scolach, palamour d'ho ravach ; pa ziffenn outhô, hini mwy ne zistrô da scrabat erw ${ }^{76}$.

Le peuple est persuadé que depuis ce temps là les gwelans ne sauroient nuire, que même le contact de la terre franche leur brûle les pieds ; ce qui est cause qu'ils ne peuvent guères s'y arrêter ${ }^{77}$.

De même, à propos du verbe «unia», tombé en désuétude, il écrit : «Il est vrai que les prédicateurs qui ne se piquent guères de parler correctement, et quelques autres à leur exemple, disent souvent unissa pour unir, de même que finissa pour finir ${ }^{78}$.» Ce breton d'Église est donc appauvri, tout comme le breton des villes. Dans sa longue présentation des différentes lettres de l'alphabet breton, il fait cette remarque sociolinguistique très fine à propos du breton parlé par les citadins :

La lettre $\mathrm{R}$ peut bien avoir été remplacée par les Bretons dans quelques mots de nouvelle création qui etoient étrangers à leur langue et qu'ils auront assez mal entendus pour lui substituer L tels sont Prioldi, Ploculeur, et Ral ; mais ce dernier n'est guères adopté que dans les villes où la langue est plus altérée, car ceux qui sçavent le Breton disent Rouez qui signifie Rare ${ }^{79}$.

À propos du terme «fallentez», il rapporte des paroles qui, si elles sont authentiques, n'ont pu être produites que par un commerçant - de Morlaix, sans doute, ville qu'il fréquentait assidûment ${ }^{80}$.

On s'en sert également pour exprimer le peu de valeur, la dépréciation ou le vil prix des denrées, vilitas. C'est encore un

76. «À la façon de moutons, il conduit les goélands / À son maître, au collège, à cause de leurs dégâts : / Puisqu'il le leur interdit, plus aucun d'entre eux ne retourne / Gratter le sillon».

77. III, 1051-1052.

78. VIII, 869.

79. I, XXXVI-XXXVII

80. Voir Louis DUJARDIN, «Un lexicographe breton ignoré : Pierre-Joseph-Jean Coëtanlem de Rostiviec (1749-1827)», Nouvelle Revue de Bretagne, janvierfévrier $1948, \mathrm{n}^{\circ} 1$, p. 42-48. 
dérivé de fall. Voici une phrase très ordinaire chez nos colons quand le commerce n'allait pas à leur gré : Kemment a fallentez a voa war an traou ma teuenn scañ-bras d'ar ghear, mot à mot : il $\mathrm{y}$ avoit tant de foiblesse sur les choses que je venois grandement léger à la maison, c'est-à-dire : Les denrées se vendoient à si bas prix que je m'en retournois très-légerement à la maison ou la bourse vuide, ce qui revient aux plaintes du berger de Mantoue :

Non unquam gravis ære domum mihi dextra redibat ${ }^{81}$. Virg. Bucol. Eglog. 1. p. $6^{82}$.

De même, dans un développement sur les caractéristiques du dialecte de Léon et la prononciation / $/$ r/ de l'article indéfini, il laisse entendre qu'il y a une pratique défectueuse du breton parmi les gens de justice - alors qu'il s'agit, en fait, d'un archaïsme montrant, davantage que de l'ignorance, une culture certaine de la langue écrite ancienne.

Je conviens au surplus que la pluspart des noms propres sont défigurés dans les actes. Les gens de justice qui les rédigent demeurent ordinairement dans les villes : ils ne savent pas tous le breton ; la pluspart le prononcent très-mal : ils négligent ordinairement les aspirations et les variations des mutes, et si on trouve si souvent An pour Ar dans les noms propres, cela vient surtout de l'ignorance des suppôts de la Chicane, qui fourrent leur An partout ${ }^{83}$.

C'est un paysage sociolinguistique assez complexe que Coëtanlem dessine, en creux, dans son grand œuvre. Pour lui, il y a une très nette distinction entre des sources écrites - latines et françaises - et des sources bretonnes - uniquement orales.

$*$

Se demander pourquoi Coëtanlem ne s'appuie que sur des sources bretonnes orales oblige tout d'abord à reconnaître qu'il confirme ce que l'on sait de l'histoire sociolinguistique du breton. Mais une première explication s'impose : pour Coëtanlem, les textes bretons des siècles passés sont fautifs.

81. «Jamais je ne revenais au logis les mains chargées d'argent».

82 . III, 340.

83 . I, LX. 
Et malgré ma vénération pour l'antiquité, je soupconne que les écrits Bret. qui sont venus jusqu'à nous, en très petit nombre, n'etoient pas fort corrects. Les doubles ff par lesquelles les anciens écrivains affectoient de terminer leurs infinitifs ne signifient rien en Léon où on ne les prononce pas du tout, et où l'on a très bien fait de les supprimer, puisqu'on n'en tient aucun compte ${ }^{84}$.

Logiquement, la littérature bretonne n'a pour lui aucune valeur, ni même aucune existence - «Il seroit bien difficile de trouver dans cette langue un ecrit de 500 ans seulement. Seroit-on fondé làdessus à dire qu'elle n'a pas 500 ans d'antiquité ${ }^{25} \gg$. Dans son dictionnaire, Le Pelletier cite la préface d'un vieux catéchisme, imprimé en 1623, et dans lequel il lit «calz a traëzou». Coëtanlem précise alors :

Mais en attendant, je crois qu'on n'a pas malfait de laisser tomber en désuétude, comme inutile, et propre seulement à causer des equivoques, le pl. Traezou du vieux catéchisme cité par D.P. qui est d'ailleurs incorrect, ainsi que la pluspart des livres bretons qui sont parvenus jusqu'à nous, quoique leur nombre ne soit pas considérable ${ }^{86}$.

Cette représentation de l'écrit breton explique que son dictionnaire nous peigne le paysage sociolinguistique dans lequel il évolue. Au XVIII ${ }^{\mathrm{e}}$ siècle, à côté des badumes, réservés à l'oral, il existe d'autres registres de bretons : le breton d'Église - façonné depuis le $\mathrm{XVI}^{\mathrm{e}}$ siècle au moins ${ }^{87}$-, le breton des villes - dont la publication des colloques, au début du XVII ${ }^{\mathrm{e}}$ siècle, est un bon témoignage ${ }^{88}$-, et le breton mondain. À l'époque où Coëtanlem rédige ses pages, ces deux derniers registres du breton cessent peu à peu d'être efficients. Le breton mondain n'a produit que des œuvres restées à l'état de manuscrit et son contenu parfois libertin n'a plus de public - la langue polie des salons est devenue suspecte et a dû céder devant les exigences de la nouvelle langue politique

84. II, 377, sous «coenvi».

85. II. 525-526 sous « coust».

86. VIII, 511, sous «traezou».

87. Voir Yves LE BERRE, Entre le riche et le pauvre. La littérature du breton entre 1450 et 1650, Brest, Emgleo Breiz, 2012.

88. Voir Dictionnaire et colloques francois et breton. Traduits du François en Breton par G. Quicquer de Roscoff, imprimé à Morlaix en 1626. 
de la Révolution ${ }^{89}$, avant de rompre devant le paysanisme français de langue bretonne véhiculé bientôt par l'Église - ; le breton des villes a surtout relevé du domaine de l'oral, servant à la bourgeoisie morlaisienne, notamment, à se distinguer des paysans.

Mais, pour être entendue, l'entreprise encyclopédique de Coëtanlem doit également être lue au regard de ses opinions politiques telles qu'elles transparaissent à travers les pages du dictionnaire. Voici un extrait du second volume, sans aucun doute rédigé avant 1814.

Nous sortons d'une Révolution qui a pensé bouleverser toute l'Europe. Nous avons vu les thrônes renversés, les autels détruits, des classes entières de citoyens proscrites, les propriétés envahies, les loix sans vigueur, le désordre et l'anarchie étendant partout leurs ravages. Je ne pousserai pas plus loin ce tableau hideux, mais personne n'ignore que pour arrêter ce torrent qui menaçoit de tout engloutir, il ne falloit pas moins qu'un héros suscité par la providence même, et Napoléon a paru. De la même main dont il a relevé le thrône des françs, il a commencé par relever les autels, rétablir la religion, rappeller ses ministres et leur rendre tous les biens non-aliénés dont on les avoit dépouillés.

Maintenant si, sous prétexte de célébrer tant de bienfaits, il se trouvoit un prêtre assez lache et assez impudent pour avancer que l'Eglise de France seroit aujourd'hui bien pauvre, si elle n'avoit point d'autres revenus que ceux que lui ont donnés les prétendus rois ou empereurs, $\mathrm{S}^{\mathrm{t}}$ Louis, Charlemagne et autres, de quel oëil ce héros magnanime recevroit-il un hommage si méprisable ? Quelle opinion auroit-il de l'esprit, du jugement et surtout de la gratitude d'un si vil adulateur ? ${ }^{90}$

$\mathrm{Au}$ septième volume, rédigé après 1817 , le portrait de Napoléon n'est plus le même : «La nation espagnole a donné les preuves les plus éclatantes de son courage et de son dévouement pendant la lutte terrible qu'elle a eu à soutenir contre Bonaparte, le tyran de l'Europe ${ }^{91}$.» Pourtant, entre ces deux citations, il n'y a pas nécessairement contradiction. Napoléon a été chassé du pouvoir et la

89. Voir Antoine LiLti, «Mondanité et Révolution...», op. cit., p. 47.

90. II. 197 sous «castell».

91. VII, 601, sous «spaign». 
Restauration est sans doute une divine surprise pour Coëtanlem. Mais, à ses yeux, l'épisode napoléonien a certainement été salutaire. En effet, Coëtanlem a connu la fin d'un monde qu'il regrette ${ }^{92}$ et le rôle d'intermédiaire entre le peuple et l'État qu'il n'avait sans doute pas manqué de tenir, sous l'Ancien Régime, a été mis à mal par la tourmente révolutionnaire. Cependant, Napoléon a réussi l'alliance entre droite et gauche : l'autorité du militaire, c'est la Droite ; l'appui sur l'universalité du peuple, via le plébiscite, c'est la Gauche. De même, le Concordat - que Coëtanlem vante dans le premier extrait supra - n'est ni une réaction, ni une révolution : l'Église et l'État sont sur un pied d'égalité ${ }^{93}$. Le Concordat a donc pour conséquence d'établir la paix avec les catholiques et de priver les monarchistes de l'essentiel de leurs troupes. Autour de la personne de Napoléon et de sa politique, il y a bel et bien une alliance du local et de l'universel : un centrisme par addition des extrêmes ${ }^{94}$.

La longue remarque que le sieur de Coëtanlem fournit dans le sixième volume, sous l'article «Roue», est très révélatrice de sa culture politique - elle permet d'ailleurs de dater la rédaction de ce volume après 1815. Il met en lumière le grand rapport qu'ont ensemble, selon lui, les noms «Douë / Dieu», «Rouё / Roi» et «Rouen̂v / Rame», «tant pour le son que pour le sens»:

En effet Dieu qui a crée ce vaste univers par sa toute-puissance est le même qui le régit, qui le conduit, qui le gouverne par sa sagesse ; et c'est ce Roi des Rois, cet être suprême, infini,

92. À propos du domaine congéable, il écrit par exemple : «Mais les loix qui ont été faites, pendant le cours de la révolution, sur tout ce qui concernoit les biens de cette espèce, les a dénaturés et avilis. La manière énergique avec laquelle le citoyen Cambri s'est exprimé à ce sujet, dans son voyage du Finistère, auroit bien pu mériter les honneurs de la guillotine à tout autre qui en eut parlé avec tant d'irrévérence ; son civisme l'en préserva ; mais comme ce morceau est peutêtre ce qu'il y a de plus juste et de plus vrai dans tout son ouvrage, je me fais un plaisir de le transcrire ici [...] Tous les honnêtes gens partagent l'indignation de l'auteur contre ce décret inique [27 août 1792], dont les effets subsistent toûjours en grande partie» (III, 1233-1234, sous «gwir»).

93. Voir Fabrice Bouthillon, «Ceci n'est pas un sacre», Commentaire, numéro 133, printemps 2011, p. 129-134.

94. Voir F. BouthiLlon, L'illégitimité de la République. Considérations sur l'histoire politique de la France au XIXe siècle (1851-1914), collection Commentaire, Plon, 2005. 
éternel, immuable, que nous appelons Doue, Dieu ; et nous faisons profession de n'adorer que lui seul. C'est par sa grace, Dei gratiâ, que le souverain auquel il a confié son autorité temporelle, nous régit, nous conduit et nous gouverne. Per me reges regnant, et legum conditores justa decernunt ; Per me principes imperant, et potentes decernunt justitiam. Proverb. 8. $15^{95}$. Et puisque c'est Dieu même qui a établi le monarque, nous lui devons respect, obéissance et fidélité ; et c'est à cet oint du seigneur, que nous révérons comme son image et son lieutenant, que nous donnons le nom de Roue, Roi. Enfin, la rame ou l'aviron est l'instrument avec lequel les marins conduisent, régissent et gouvernent leurs bateaux et leurs barques, et c'est cet instrument que nous appellons Rouen̂v, qui diffère si peu de Roue, Roi, qu'on peut croire que c'est le même mot dont on a prolongé le son final, uniquement pour distinguer ses acceptions différentes ${ }^{96}[\ldots]$

Le Roi est le représentant de Dieu sur terre et il est le Père de ses sujets :

Dans le même tome du Traité de l'opinion ${ }^{97}$, pag. 81, l'auteur prouve par l'authorité des anciens, que les hommes en formant les premières sociétés, se sont soumis au gouvernement monarchique, à l'imitation de la puissance paternelle, et soutient que le gouvernement monarchique est celui de tous où il se trouve plus de liberté. Nos Républicains françs. en avoient d'abord jugé tout autrement, puisqu'ils avoient juré haîne aux Rois et à la Royauté ; et que dans leur première ferveur ils avoient ajoûté à ce serment celui de vivre libres ou de mourir; mais au bout de quelques années d'epreuves, leur enthousiasme s'est refroidi : ils ont abjuré leur erreur, et reconnu que la vraie liberté n'existoit nulle part avec plus de latitude et d'agréments que dans un païs gouverné par un monarque equitable et pieux ; c'etoit aussi le sentiment exprimé il y a plus de quatorze cents ans, avec autant de noblesse que d'energie par le plus fameux Poëte latin qui aît paru depuis Virgile. Les voici :

95. «Par moi les rois règnent, et les princes ordonnent ce qui est juste. / Par moi dominent les puissants et les grands, et tous les juges de la terre».

96. VI, 1002.

97. Il cite le tome $5 \mathrm{du}$ Traité de l'opinion ou Mémoires pour servir à l'histoire de l'esprit humain, de Gilbert-Charles Le Gendre publié en 6 volumes à compter de 1735. 
Fallitur egregio quisquis sub principe credit servitium : numquam libertas gratior extat quam sub rege pio ${ }^{98}$.

Claudian. De laudib. stilic. Lib. 3. V. $113^{99}$.

La subordination du politique au religieux est donc toute naturelle et, heureusement, elle n'a pas été totalement mise à mal par les Républicains, à preuve la tentative avortée de réformer le découpage de la semaine en sept jours :

Mais nos Républicains, qui, sous prétexte de tout régénerer, firent leurs efforts pour tout détruire, tentèrent par un Décret de la Convention daté du $44^{\mathrm{e}}$ jour de frimaire, l'an $2^{\mathrm{e}}$ de la République française une et indivisible, de reformer totalement le calendrier et l'organisation de l'année ; en conséquence, sans égard à l'usage antique, vénérable et général de partager le mois en semaines, de sept jours chacune, ils le partagèrent en trois décades, ou trois dizaines de jours, dont ils changèrent également les noms. Le but manifeste de tous ces changements étoit d'abolir le dimanche ou le jour de repos consacré au Seigneur, qui reparoissoit régulièrement chaque septieme jour au bout de la semaine ; mais en dépit de leur jactance, ces hardis novateurs ont vu renverser en peu de temps leurs loix insensées : la semaine n'a pas tardé à remplacer de nouveau la décade ; et le dimanche a bientôt triomphé du décadi comme cela devoit être ${ }^{100}$.

Bien entendu, c'est cette subordination du politique au religieux, cet augustinisme politique à la mode de Bretagne, qui explique le paternalisme dont ne sont pas exemptes certaines de ses remarques ou de ses leçons de morale. En effet, on l'a vu, le sieur de Coëtanlem connaît fort bien le breton et il fréquente régulièrement ses paysans ${ }^{101}$. Il a même des informateurs attitrés. Ainsi, à propos du

98. «Quiconque pense qu'il est en esclavage lorsqu'il sert un prince éminent est dans l'erreur : la liberté n'est jamais plus grande que sous un roi pieux» (extrait de l'Eloge de Stilicon de Claudien).

99. VI, 1007-1008, sous «roue».

100. VII, 382-383, sous «seisun».

101. «J'ai entendu aussi les cultivateurs de ce païs se servir de sea; mais en parlant à leurs bestiaux seulement ; ce qui me fait croire que ce n'est autre qu'un terme de jargon» (VII, 359, sous «sea», que DP présente comme un impératif pour dire «doucement; patience»). 
mot «reiz», se pose-t-il la question de savoir si ce nom est masculin ou féminin :

Je penchois aussi à le faire mascul. mais ne voulant pas m'en rapporter tout-à-fait à mon propre jugement, j'ai consulté un cultivateur qui parle assez bien, et je lui ai demandé si on disoit Daou Reiz ou Diou Reiz, il m'a répondu sans hésiter qu'on disoit Daou Reiz, d'où j'ai conclu que je ne me trompois pas, et que, sur ce point, on devoit préférer le sentiment du P.G. quoiqu'il soit bien loin d'etre infaillible, à celui de D.P. qui n'etant pas Breton d'origine, étoit moins sûr de la véritable prononciation, quoique beaucoup plus habile homme ${ }^{102}$.

Il connaît donc très bien le terrain breton et l'anecdote suivante le confirme - elle témoigne également d'une certaine forme de condescendance. Sous l'article «caezrell», il explique que la belette a plusieurs noms, par superstition. Il faut éviter de l'appeler par son vrai nom, de peur qu'elle ne vienne :

Quoiqu'il en soit je conviens que cette superstition subsiste encore dans nos campagnes, et de là vient sans doute que ces petits animaux ont tant de noms différents ; car avec le temps la premiere Epithete passant pour le vrai nom, qu'on veut s'abstenir de prononcer, on leur en impose un nouveau. Pour ce qui concerne la Belette je n'en sçaurois douter, après avoir eu occasion de m'en convaincre bien clairement. Je me trouvois un jour dans une ferme du côté de Tréguier, où l'on me dit, en parlant des vaches que l'on craignoit que les Demoiselles ne se fussent introduites dans l'etable. J'étois jeune alors, et dès qu'il fut question de demoiselles, je témoignai le désir de faire connoissance avec elles, mais la vieille fermière me tira de mon erreur en m'apprenant que ce qu'elle avoit désigné par le nom de Demesellet (Demoiselles) n'etoit autre chose que de petits animaux, qu'elle nommoit ainsi, parceque s'ils s'entendoient appeler de leur vrai nom, ils ne manqueroient pas de venir teter ses vaches, et les épuiseroient de manière à les faire périr. Je ne pus m'empêcher de rire de ma méprise et de sa superstition; et je lui demandai quel étoit le vrai nom de ces redoutables animaux, puisque Demesell (Demoiselle) pl. Demesellet, n'etoit qu'une affaire d'etiquette, un simple titre de politesse que prescrivoit la

102. VI, 803. 
crainte. Elle me répondit tout bas à l'oreille que le nom de ce petit animal etoit Coantic, pl. Coantighet. C'est un diminutif de Coant, joli, jolie et signifie par conséquent petite jolie ou joliette il y a assez d'apparence que ce dernier nom est encore une Epithète aussi bien que Buhan, Cäezrell, Propic ${ }^{103}$.

Comme un père - ou un grand-père -, il est donc à même de distinguer ce qui est bien de ce qui est mal, et de laisser entendre ce qui est bon pour la vie en société : les hommes volages sont condamnés car porteurs de la grosse vérole ${ }^{104}$; les méfaits du tabac sont mis en lumière ${ }^{105}$; les mesures d'hygiène élémentaire sont présentées ${ }^{106}$; les moqueurs sont vilipendés ${ }^{107}$; la gourmandise est peinte comme un vice $^{108}$; la jalousie est dénoncée ${ }^{109}$; la veuve est défendue face aux attaques dont elle fait souvent l'objet, parce que l'on doit des égards au «sexe charmant ${ }^{110}$ »; la retenue et la modestie «qui conviennent plus particulièrement» à ce dernier sexe sont rappelées ${ }^{111}$. Enfin, très régulièrement, Coëtanlem dénonce la superstition qui règne sur les campagnes.

Nos païsans y attachent aussi une idée de quelque chose de surnaturel, et plusieurs se persuadent volontiers qu'un ouragan, par exemple, qu'ils appellent Eur bar amser, est causé ou excité par le diable ou par quelque sorcier. Fort souvent ils imputent encore aux sorciers les maladies inconnues ou extraordinaires dont ils sont attaqués, d'autres fois à l'air ou au vent infecté par des sortileges, et quelquefois cependant pour avoir passé sous le vent d'une marre infecte ou qui contenoit de l'eau croupie. Les espèces de mages ou soidisant médecins auxquels ils ont recours

103. II, 23-24, sous «caezrell».

104. I, 552, sous «brec' $h »$.

105. I, 672, sous «butun».

106. Par exemple, à propos de la teigne : «Il est certain que ce nom se s'applique point à d'autre mal qu'à cette petite gale ou eruption cutanée qui vient principalement à la tête des enfants, et qui est comme naturelle à presque tous ceux de ce païs» (VIII, 384, sous «tocken»).

107. «Il n’y a point de caractère si dangéreux que celui d'un railleur» (III, 701, sous «goap»).

108. III, 838, sous «gourmant».

109. I, 66-67, sous «amezec».

110. IV, 304.

111. VIII, 999, sous «yourc' $h »$. 
leur font ordinairement l'application de quelques simples, qu'ils appellent Lousou ou leur en font avaler le jus, en y melant un peu d'eau benite, ou marmotant quelques paroles barbares ou magiques, (car la pluspart passent aussi pour magiciens,) et quelques cérémonies superstitieuses ou ridicules. Lorsque les malades en reviennent, ces charlatans ne manquent pas de s'en attribuer la gloire, et leur réputation s'accroît de plus en plus. Si les malades périssent, on se contente de dire : Scoet e oant gant bar. Ils étoient frappés ou atteints ou attaqués non seulement d'un mal subit ou violent, mais même surnaturel ou d'un maléfice : ou bien Scoet e oant gant drouc-avel, à la lettre, ils étoient frappés ou atteints par mauvais vent; et on a vu plus haut ce qu'ils entendoient par mauvais vent. Une telle déclaration de la part de ces jongleurs les justifie pleinement aux yeux d'une populace crédule, parcequ'ils ont la modestie d'avouer que leur science ne peut pas toujours triompher des maladies qui proviennent d'une cause surnaturelle ${ }^{112}$.

Les paysans sont donc de grands enfants - «on ne doit pas être surpris de la prévention qui subsiste encore chez le menu peuple ignorant et crédule et toujours ami du merveilleux ${ }^{113} \gg-$, empreints pourtant d'une certaine noblesse - «Quelques grossiers que soient les gens du peuple, en Bretagne comme ailleurs, ils ont cependant une certaine retenue, même dans leurs emportements, en sorte que dans les imprécations, les jurements, les termes injurieux ou blasphématoires dont ils se servent, ils ont ordinairement le soin de déguiser ou de mutiler les commencement ou la fin des mots, pour ne pas dire les choses trop crûement. J'ai souvent entendu se servir du terme de Mastokin, et il m'a paru qu'on n'y attachoit guères que le sens de vilain malotru, vilain poliçon, ou vilain gueux; mais je soupçonne que c'est pour Mastokan ou Masdogan, qui signifie proprement vilain cocu, dont on aurait à dessein déguisé la finale pour la raison que je viens d'alléguer ${ }^{114} \gg$-, et gardiens d'une connaissance bretonne dont ils n'ont pas même conscience. Coëtanlem est le grand-père qui

112. I, 272, sous «bar». Voir aussi, dans le même volume (411), son attaque contre les charlatans et les sorciers, répétée dans le volume VII (560, sous «sorça»).

113. I, 491-492, sous «boudet-ew». "Au reste le peuple qui croit aux sceptres, aux lutins, aux esprits follets, les suppose aussi animés» (VIII, 144, sous «tasmant»). 114. V, 197, sous «mastokin». 
transmet ce savoir, pour la postérité, et qui contribue à l'invention d'un patrimoine ethnographique ${ }^{115}$, archaïque et enchanteur. Le dictionnaire peut alors être lu comme une expression et un concentré de la culture sociale et politique de son auteur qui, logiquement, refuse dans le même temps le légendaire et l'esprit des philosophes «qui à force de raisonner sur tout sont parvenus à douter à peu-près de tout ${ }^{116} \gg$.

$*$

Mais ce grand œuvre est donc aussi, et peut-être surtout, une encyclopédie qui allie anecdotes et références livresques, oral et écrit, bretons, français et latin, local et universel : d'une certaine façon, Coëtanlem réalise une addition des extrêmes. Et c'est cette addition qui explique en partie sa celtomanie ${ }^{117}$ : le prestige magique des origines attribué aux Celtes antiques permet au sieur de Coëtanlem de compenser la perte de prestige - symbolique ou matérielle, peu importe - subie suite aux troubles révolutionnaires.

Ce dictionnaire est une source d'informations sur les habitudes culturelles et langagières des Bas-Bretons de la fin du XVIII et du début du XIX ${ }^{\mathrm{e}}$ siècle, mais c'est aussi un témoignage de première main sur la culture d'un noble campagnard porteur de multiples connaissances, témoin d'une époque révolue et acteur - même passif - de bouleversements sociaux et politiques considérables. Car Coëtanlem allie aussi modestie et prétention ; religion catholique et Lumières - tamisées, certes - ; celtomanie et goût pour la connaissance ; ironie et condescendance.

C'est cette situation d'entre-deux qui se laisse entendre admirablement dans cette remarque, à la suite de l'article «Cac' $h »$ :

Comme il y a mille occasions où l'on se trouve obligé d'appeller les choses du nom qui leur est propre ; au lieu des

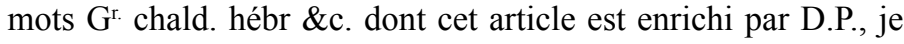
me permettrai de m'expliquer en $\mathrm{f}^{\text {': }}$ ou en bret. Cac'h n'est point

115. Voir Odile PARSIS-BARUBÉ, La province antiquaire. L'invention de l'histoire locale en France (1800-1870), Éditions du CTHS, 2011, p. 55 sq.

116. VII, 933, sous «sul».

117. Voir quelques exemples dans «Les mots et les causes», supra. Sur la celtomanie, voir Jo Rio, Mythes fondateurs de la Bretagne. Aux origines de la celtomanie (éditions Ouest-France, Rennes, 2000) et O. PARSIS-BARUBÉ, op. cit. 
chez nous l'excrémement [sic] même qu'on appelle merde. Cet excrément se nomme Cauc'h, qu'on prononce en Léon Caoc'h, dissylabe, et que D.P. écrit ci-après Coc'h, parcequ'on en fait un monosyllabe hors de Léon. Ainsi Davies a été induit en erreur lorsqu'il nous a prêté Cac'h en ce sens, mais peut-être a-t-il voulu écrire Cawc'h, que je crois le plus exact. Cac'h est le chiement, s'il m'est permis de créer un terme nouveau, ou l'action même par laquelle on chie. [...] Ce seroit offenser un lecteur délicat que de m'etendre davantage sur cette matière. Il ne paroîtra que trop long à un censeur sévère, qui ne manquera pas de blâmer mon chiement, mais au lieu de demander grâce pour cet avorton forgé sous de malheureux auspices, je suis de si bonne composition que je consens qu'il soit proscrit dès sa naissance et qu'on lui substitue si l'on veut, cette circonlocution honnête : l'expulsion des matières fécales. Comme tout le monde n'a pas le goût si difficile, j'aurois transcrit ici en faveur des amateurs... de la poësie française deux odes assez bien faites sur la nécessité d'expulser ces matières; et pour leur donner un avant goût de ces précieux morceaux, je les aurois fait précéder d'une énigme facile à deviner. Je leur reservois pour la bonne bouche un logogriphe analogue au sujet, mais la critique me fait peur, et je ne leur donnerai pour tout potage que cette epigramme lat. puisque le latin ne put pas.

Ventris onus misero (nec te pudet) excipis auro :

Basse bibis vitro, carius ergo cacas ${ }^{118}$. Martial ${ }^{119}$.

118. Livre I, 37 : «Cela ne te gêne pas de faire dans un plat d'or (pauvre plat lorsque j'y pense), tandis que tu bois ton vin dans une coupe de verre. J'en conclus, mon cher Bassus, que ta merde coûte cher» (traduction de Pierre Trouiller).

119. II, 12-13. 


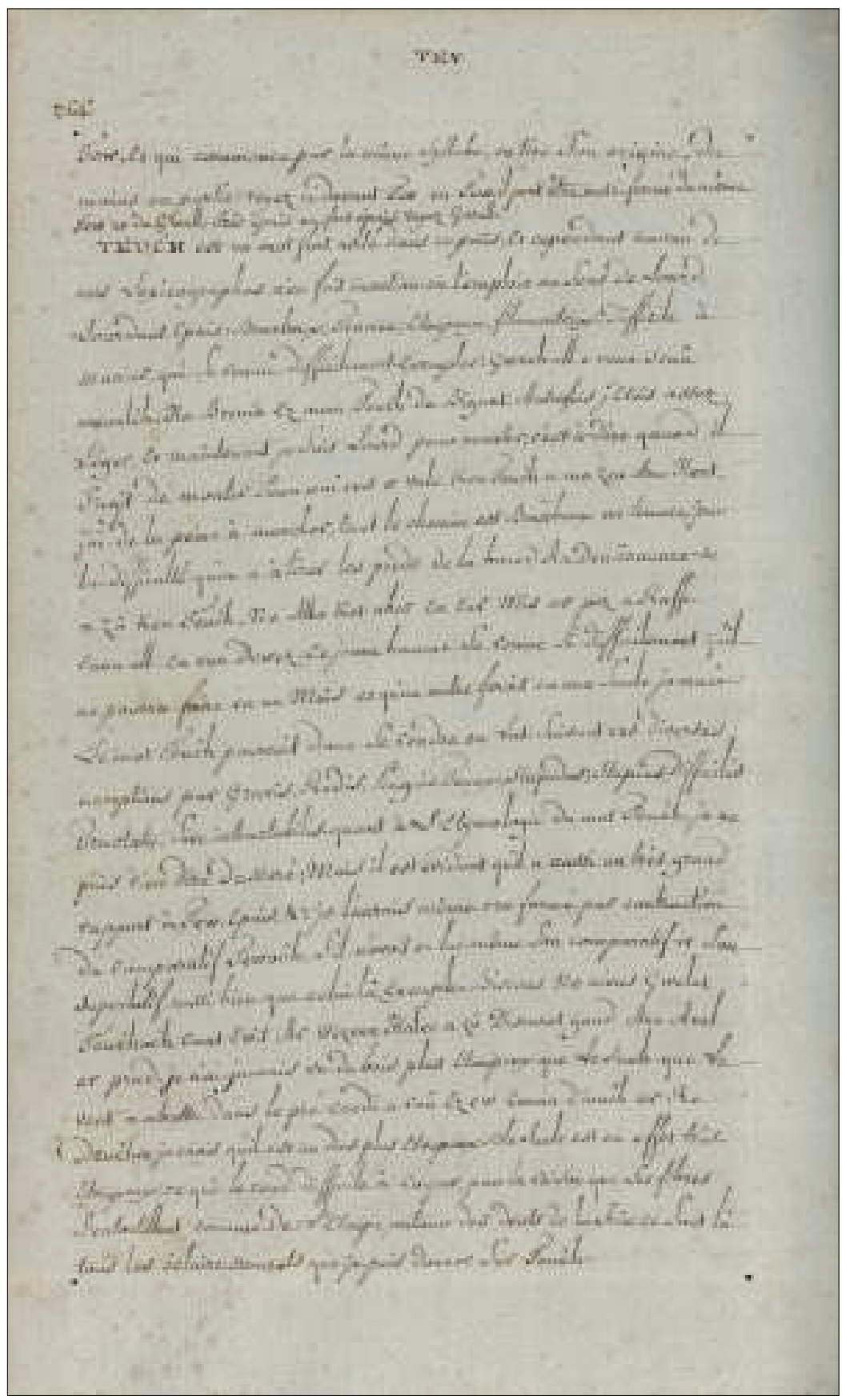

